\title{
Improving the energy efficiency of the pipeline system by ensuring its linear flow characteristic
}

\author{
Denis Dymochkin ${ }^{1}$, Mariya Kilina ${ }^{*}$, Vyacheslav Grishenko ${ }^{1}$, and Alexandr Kharchenko ${ }^{2}$ \\ ${ }^{1}$ Don state technical university, Department "Hydraulics, hydropneumoautomatics and heat \\ management”, 344000, Gagarin square 1, Rostov-on-Don, Russian Federation \\ ${ }^{2}$ Camozzi Pneumatic LLC, Educational and Scientific Department, 141592, Attilio Camozzi Street 1, \\ Chasnikovo, Solnechnogorskiy District, Moscow region, Russian Federation
}

\begin{abstract}
Recently, SMART positioners have been widely used to control the on-off and control valves. These devices allow you to set an arbitrary user dependence of the valve travel on the control signal. This makes it possible to provide a linear throughput for the entire piping system. This allows the use of smaller type control valves while maintaining the control range. Accordingly, the energy consumption for controlling the shutoff element is reduced; the value of the reinforcement energy efficiency indicator is improved. In that work analytically calculates a user dependency that takes into account the value of pipeline throughput and allows to obtain a linear throughput of the pipeline system in the entire range of control signal change.
\end{abstract}

\section{Introduction}

The introduction of modern automation technologies improves the energy efficiency of pipeline valves and pipeline systems. Automatic control systems make it possible to optimize operation modes of pipeline systems, which leads to significant savings of energy resources [1]. The optimization criterion can be a minimum of energy, time, or material consumption.

In many modern industries, on-off and control valves are used to automatically control the flow of liquid and gaseous process media. Linear and rotary pneumatic actuators are often used to control valve travel. An important area of research in this area is improving the positioning accuracy of pneumatic actuators. To solve this problem, it is necessary to study the dynamic characteristics and search for the optimal structure of the drive [2-4].

Other directions of energy efficiency improvement are search of new materials and technologies of reinforcement production [5], development of criteria of energy efficiency assessment [6].

However, the static characteristics of the drive are also important. It is desirable that they be linear. This simplifies the creation of an automatic control system, allows you to make control more accurate. If pressure or flow rate of process medium during control exceeds the required value, its release is performed. The product of pressure per flow rate is

\footnotetext{
*Corresponding author: mariya-kilina@yandex.ru
} 
the flow power. Therefore, the unproductive discharge of the process medium leads to energy losses. Thus, increasing control accuracy due to linearization of static characteristics of control valves increases the energy efficiency of pipeline systems. The paper proposes a solution to this problem.

\section{Methods and materials}

One of the main characteristics of such valves is the flow characteristic - the dependence of the flow capacity $\mathrm{K}_{V}$ on the stroke of the valves [7]. It is easier to create an automatic control system if the valves have a linear throughput:

$$
F(h)=F_{0}+m \cdot h=F_{0}(1-h)+h
$$

where $F(h)$ is relative flow capacity, $F_{0}$ is the initial relative throughput, $m$ is the proportionality coefficient (at relative stroke $h=1$ the relative throughput $F=1$, and $\left.m=1-F_{0}\right)$, h is the relative stroke ratio.

The flow characteristic of the pipeline system is determined both by the characteristic of the valves and by the throughput of the pipeline. If the flow capacity of the valves exceeds the pipeline capacity by more than three times, the system capacity becomes substantially non-linear [8]. In this case, valves with equal percentage flow characteristic are used:

$$
F(h)=F_{0}{ }^{l-h}
$$

In this case, the flow characteristic of the system is close to linear on the $50 \ldots 70 \%$ stroke of the valves.

Isolation and control valves are controlled using positioners. Positioner provides linear relationship between control signal and control valve stroke. Modern SMART positioners allow you to additionally program the user's dependence of the valve travel on the control signal, which is equivalent to the introduction of a corrective link. A wide range of SMART positioners is currently being issued, for example, as presented in [9]. Correction link is one of the methods of obtaining linear static characteristics in automatic control systems [10].

Programming of the user flow characteristic can allow to obtain a linear throughput characteristic of the system "control valves - pipeline" at the entire range of valve travel. The main task is to determine the type of this characteristic (that is, the static transfer function of the correction link).

\section{Results and discussion}

The known formulae [11] are used to determine the flow resistance coefficient $\xi$ and the flow characteristic $K_{V}$. From these, a unique relationship between $\xi$ and $K_{V}$ can be obtained.

Resistance coefficient of pipeline system is equal to sum of resistance coefficients of valves and pipelines. By determining the resistance coefficient of the pipeline system, we can calculate its flow capacity.

Assuming that the flow capacity of valves and pipelines differs by $\mathrm{n}$ times, an expression can be obtained to determine the flow characteristic of the pipeline system:

$$
F_{C}(h)=F(h) \cdot \sqrt{\frac{1+n^{2}}{1+n^{2} \cdot F^{2}(h)}}
$$


where $F_{C}(h)$ is the relative capacity of the pipeline system, $\mathrm{n}$ is the ratio of the rated flow capacity of the valves to the pipeline capacity.

If we consider the driving effect $U_{\mathrm{z}}$ in relative units, then as a rule, the positioner provides a linear dependence $h=U_{\mathrm{z}}$. In this case, the programmable user function should ensure that the relative stroke $\mathrm{h}$ depends on the control signal $h=f\left(U_{\mathrm{z}}\right)$, which would ultimately provide a linear dependence on the flow capacity of the pipeline-valve system:

$$
F_{c}=F_{o c}+K_{c} U_{z}=F_{o c}+\left(1-F_{o c}\right) \cdot U_{z}
$$

where $\mathrm{F}_{0 \mathrm{C}}$ is the initial relative capacity of the pipeline system, $K_{C}$ is the proportionality coefficient (by analogy with $(1) K_{\mathrm{C}}=1-\mathrm{F}_{0 \mathrm{C}}$ ).

To find the desired user function $h=f(U z)$, equate the right parts (3) and (4):

$$
F(h) \cdot \sqrt{\frac{1+n^{2}}{1+n^{2} \cdot F^{2}(h)}}=F_{o c}+K_{c} U_{z}
$$

After simple transformations, we get:

$$
F(h)=\frac{F_{o c}+K_{c} U_{y}}{\sqrt{1+n^{2} \cdot\left[1-\left(F_{o c}+K_{c} U_{y}\right)^{2}\right]}}
$$

Substituting from (1) the function of the linear flow characteristic, after the transformations we obtain:

$$
h\left(U_{Z}\right)=\frac{F_{o c}+K_{c} U_{Z}}{\left(1-F_{0}\right) \sqrt{1+n^{2}\left[1-\left(F_{o c}+K_{c} U_{Z}\right)^{2}\right]}}-\frac{F_{0}}{1-F_{0}}
$$

Initial relative flow capacity of pipeline system is determined from formula (3). For this purpose we substitute the value of initial relative flow capacity of valves:

$$
F_{O C}=F_{0} \cdot \sqrt{\frac{1+n^{2}}{1+n^{2} \cdot F_{0}^{2}}}
$$

The user flow characteristic is given as a set of points, for each of which the value of the $\mathrm{U}_{3}$ control signal and the corresponding relative stroke value $\mathrm{h}$ are indicated. If $\mathrm{h}$ values are calculated as per formulae (7) and (8), linear dependence of flow capacity of $\Phi_{\mathrm{C}}$ pipeline system on $\mathrm{U}_{3}$ is obtained in the whole range of control signal change.

\section{Conclusion}

The graphs of the function (7) become significantly nonlinear when $\mathrm{n}$ is magnified (fig. 1).

The real technical system "reinforcement - actuator - positioner" can have significant friction forces and has characteristics such as sensitivity and hysteresis. This makes it difficult to realize the user flow characteristic at the start portions where large variations in the control signal correspond to small increments. Probably, in this case, fittings with an equal percentage flow characteristic should also be used. 


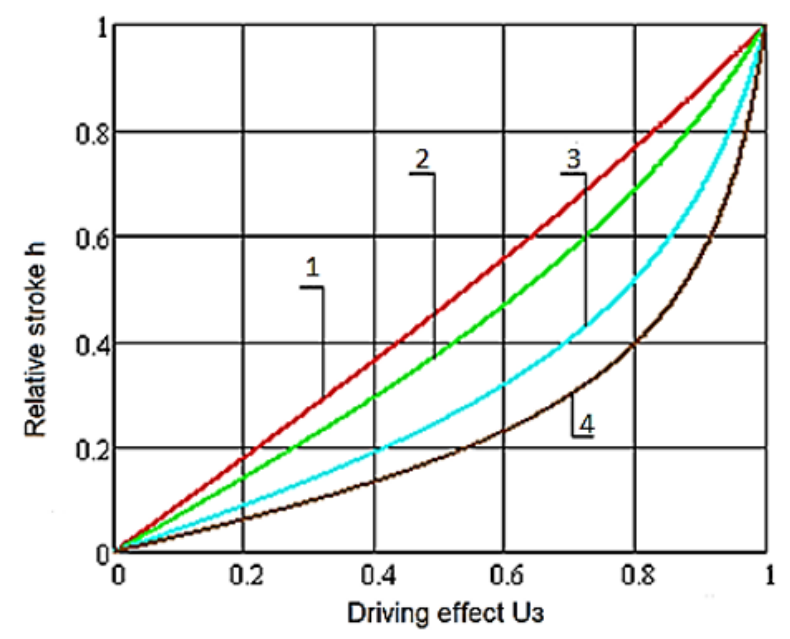

Fig. 1. User function $h=f(U z)$. 1) $F_{0}=0.04, n=0.5$; 2) $F_{0}=0.04, n=1$; 3) $F_{0}=0.04, n=2$; 4) $F_{0}=0.04, n=3$

Similarly, for valves with equal percentage flow characteristic, we obtain the following user characteristic:

$$
h\left(U_{z}\right)=1-\frac{\ln \left(F_{o c}+K_{c} U_{z}\right)-0,5 \ln \left\{1+n^{2}\left[1-\left(F_{o c}+K_{c} U_{z}\right)^{2}\right]\right\}}{\ln F_{0}}
$$

However, in this case, the situation is complicated by the fact that the inherent flow characteristic can differ significantly from the standard values, especially in the initial section with small moves. Therefore, for calculations it is necessary to use the actual flow characteristic.

The task of determining the inherent flow characteristic is discussed in part in [12]. It should be noted that the flow capacity of the valves at zero travel is equal to or close to zero. Formula (2) gives the value $\mathrm{F}_{0}$. Because of this, a discrepancy of real and standard values is obtained. Therefore, to more accurately determine the inherent flow characteristic, it is necessary to search for an alternative mathematical function for valves with an equal percentage flow characteristic.

Calculation of pipeline flow capacity can be carried out on the basis of known hydraulic formulas or using known methods and reference data [13, 14]. Programming of user characteristics is detailed in the corresponding documentation $[15,16]$.

\section{References}

1. Aksyutin O.E., Ishkov A.G., Khvorov G.A., Akopova G.S. Gas Industry, 750, 1, 52-58 (2017)

2. https://neftegas.info/upload/uf/d57/d5737a1a29ff58b1541b774bfa780eec.pdf

3. Sidorenko V.S., Korotych D.A., Grishenko V.I., Kharchenko A.N. IOP Conference Series: Materials Science and Engineering, 1029, (2020), https://doi.org/10.1088/1757899X/1029/1/012039.

4. Dao T.A., Sidorenko V.S., Dymochkin D.D. Vestnik of Don State Technical University, 15, 4, 46-53 (2015) https://doi.org/10.12737/16077, 
5. Obukhova E.N., Grishchenko V.I. Simulation of the dynamics of the positioning process of a two-way pneumatic actuator. Intelligent systems, control and Mechatronics-2018. Materials of the All-Russian Scientific and Technical Conference. 2018. pp. 165-168, Sevastopol, Russia (2018) (In Russ.):

https://www.elibrary.ru/download/elibrary_35263051_16419808.pdf

6. Poloskov S.S. Problems of weld overlay of seating surfaces of pipe fitting and solutions. Vestnik of Don State Technical University. 19,4, 349-356 (2019): https://doi.org/10.23947/1992-5980-2019-19-4-349-356

7. Dolotov A.M., Zatsarny V.A., Shpakov O.N. Systemic approach to energy efficiency of pipeline valves. Systems. Methods. Technologies, 26, 2, 66-74 (2015):

https://brstu.ru/static/unit/journal_smt/journal_smt_en/docs/number-26/66-74.pdf

8. Interstate council for standardization, metrology and certification (ISC). Interstate standard GOST 24856-2014. Pipeline valves. Terms and definitions.

9. D.F. Gurevich, Pipeline valves: Reference manual, p. 356-359 (1981)

10. Electronic catalogue. Pneumatic and Electrical Actuators, Components/Pipeline valve/ Positioners for Pneumatic Actuators and Accessories:

http://catalog.camozzi.ru/\#!d51g25s05p01 (2020)

11. V.A. Bessekersky, E.I. Popov, Theory of automatic control systems, St. Petersburg, Russia, p. 258-267 (2003)

12. Interstate council for standardization, metrology and certification (ISC). Interstate standard GOST 34437-2018. Pipeline valves. Procedure for experimental determination of hydraulic and cavitation characteristics.

13. A.V. Telepneva, D.D. Dymochkin, M.V. Telepnev, On the calculation of the hydraulic characteristics of valves, №2(17), 49-54 (2019): $\underline{\text { https://mid- }}$ journal.ru/upload/iblock/a0f/9 Telepneva_Telepnev_-Dymochkin-49 54.pdf

14. A.K. Dertsakyan, M.N. Shpotakovsky, V.G. Volkov and others, Handbook on the design of main pipelines, (1977)

15. F.A. Shevelev, A.F. Shevelev, Tables for hydraulic calculation of water pipes, (1984)

16. Smart positioner YT-3300 / 3350 / 3303 / 3301 series. Product manual: http://www.ytc.co.kr/wp-content/uploads/Data/EN/DOWNLOADS/Manual/YT3300_3350_3303_3301.pdf

17. Intelligent electro-pneumatic positioner. Series CC800, Series CC820. Manual (In Russ.): https://www.catalog.camozzi.ru/doc/positioner_cc800-820_manual.pdf 